\title{
Cervical Cancer Surveillance - Considerations in the Vaccination Era
}

\section{Georgios Michail, Georgios Androutsopoulos* and Georgios Decavalas}

Department of Obstetrics and Gynecology, University of Patras, Medical School, Rion, Greece

\begin{abstract}
Cancer of the uterine cervix is still a major cause of young women's mortality, despite more than a half a century of sustained cytological effort and the launch of the VLP HPV vaccine almost one decade ago, which was the first vaccine to target an STI. While the treatment of severe cervical dysplasias averts the development of cancer, it also represents the source of significant (mainly obstetric) morbidity.
\end{abstract}

Both incidence and mortality rates for cervical cancer vary widely across the world, with the number of deaths in low-resource settings being nearly ten times greater than in high-resource regions. The developing world accounts for almost $80 \%$ of cases. These differences reflect primarily the absence of provision of organized cervical cancer screening programs in most low-resource countries [1].The situation is different in resource-rich countries, where the majority of women who develop invasive cervical cancer have never been screened or have been inadequately screened.

The principal goal of most vaccines, including HPV vaccines is to prevent disease, rather than to prevent infection. Both VLP vaccines work by inducing neutralizing IgG serum antibodies. When the firstgeneration vaccines were launched, they mainly targeted HPV16 (known to cause approximately half of cervical cancer cases) and HPV18 (causing another fifth). At that time, the anticipated benefits from the introduction of HPV vaccination included the reduction in morbidity and costs associated with follow-up of mild or equivocal cervical lesions, decreased needs for the treatment of CIN2+ and invasive cancer and modifications to the screening program that would result in cost savings [2]. Massad et al. commented at the time that the greatest impact of vaccination on cytologic diagnosis would be a reduction in HSIL, while impact on atypical and low grade cytology would be less. Since these borderline cytologic changes make up the majority of colposcopy referrals, vaccination's impact on colposcopy resource utilization was likely to be attenuated when compared to the impact on true cancer precursors and cancer [3].

In reality, HPV vaccination has triggered vast changes in the rationale and practice of cervical screening, most important of which are the relative roles of cytology and biomarkers as screening and triage tools, alterations in the recommended ages of commencing and discontinuing screening, the appropriate screening intervals, the feasibility, role and value of primary HPV testing as a mass screening tool, and the interrelation of vaccination with cervical screening programs, in different settings [4].

Reviewing the natural history of cervical precancer, Katki et al illustrated that women aged 21-24 had almost zero cervical cancer risk, a point that underscores the low yield of screening women under age 25 and supports conservative management of women aged 2124.5 In the age group of 30-34 years, CIN2-3 lesions and stage IA cancer lesions start to accumulate within 3 years of a normal smear [6]. Screening starts to impact on fully invasive cervical cancer in women over 30 years old [7,8].It has no effect on invasive cervical cancer in the under 30 years age group, but probably has an effect on microinvasive cancer [7-9].

\author{
Publication History: \\ Received: October 25, 2016 \\ Accepted: November 10, 2016 \\ Published: November 12, 2016
}

\section{Keywords:}

Cervical cancer surveillance, Vaccination, Screening, CIN2-3 lesions, IgG serum

In an editorial dating back in 2008 addressing the theoretical dilemma of "HPV vaccination versus cervical screening", Prof Margaret Stanley pointed that both approaches are and will be needed, but implementation as a rational public health intervention will be complex and will depend upon social and economic factors [8]. Inevitably, both immunized and non-immunized birth cohorts will continue in the screening program, since the immunized group will continue to be at risk for the non-vaccine oncogenic types [8]. Vaccination plus screening should, in theory, prevent almost $100 \%$ of cervical cancers, but this could only apply in countries with organized screening programs. In populations without adequate screening, HPV vaccines at affordable cost are the only answer, since immunization as a public health intervention is highly effective, even in countries with very low resources [8]. Other authors highlight that the higher the infection rate, the more cost-effective strategies with high vaccine uptake will be and more health benefits are gained for the same cost [10]. Herd immunity is another factor that remains unaccounted for by most studies and will probably have an impact on the costeffectiveness of the vaccine, as the extent of the vaccine's efficacy is likely to have been underestimated if herd immunity occurs. Additionally, even if the vaccine effectiveness wanes, herd immunity would maintain a reasonably low infection rate, meaning that the less intensive screening scenarios will still be favored [10].

The core aim to improve the cost-effectiveness of the screening system has initially resulted in several national screening guideline committees recommending one or more of three changes: a) an increased screening interval for women who have repeatedly screened normal; b) new methods of processing cellular samples such as liquid based cytology; and c) various methods of testing for high risk HPVs [11]. Vaccination has been incorporated into the current national cervical cancer control guidelines of each country [11].

Despite no scientific body has yet endorsed such guidelines, the future of cervical cancer control may become a diversified strategy, one for non-vaccinated birth cohorts and another for vaccinated

*Corresponding Author: Prof. Georgios Androutsopoulos, Department of Obstetrics and Gynecology, University of Patras, Medical School, Rion 26504 Greece, Tel: +306974088092; E-mail: androutsopoulos@upatras.gr

Citation: Michail G, Androutsopoulos G, Decavalas G (2016) Cervical Cancer Surveillance - Considerations in the Vaccination Era. Int J Community Fam Med 1: 117. doi: https://doi.org/10.15344/2456-3498/2016/117

Copyright: () 2016 Michail. This is an open-access article distributed under the terms of the Creative Commons Attribution License, which permits unrestricted use, distribution, and reproduction in any medium, provided the original author and source are credited. 
Citation: Michail G, Androutsopoulos G, Decavalas G (2016) Cervical Cancer Surveillance - Considerations in the Vaccination Era. Int J Community Fam Med 1: 117. doi: https://doi.org/10.15344/2456-3498/2016/117

Page 2 of 3

cohorts [12]. Other authors advocate discriminations depending on the setting: Highly-resourced countries, with established cytology screening have usually reduced their cervical cancer rates [13]. Most have successfully instituted vaccination for female adolescents and catch-up programs for older teens. The authors point that as more cohorts are vaccinated and start reaching the screening age, HPV vaccination will affect screening positivity, with a direct negative effect on the performance of cytology. This will inevitably lead to a gradual change from cytology to HPV screening over the next decade. The concept of "HPV faster" represents another radical strategic option [14]. In less-resourced countries where cervical cancer is an extremely common cancer, cytology screening has been used opportunistically for several decades without real effect on cervical cancer rates [13] Ideally, as in more resourced settings, a combination of vaccination in young women and screening of those older than 30 years should be implemented but financial constrains are severe. The use of VIA and other visual techniques have been proposed, but those are largely subjective and expertise-dependent. Furthermore, since HPV type 16 is related to more clear visual acetowhite changes in the epithelium, a reduction of the performance of VIA for cervical cancer screening to identify women with CIN2 is anticipated in vaccinated populations [15]. For the above reasons, in less-resourced countries HPV vaccination seems a more viable option.

For both vaccinated and unvaccinated women, age-based screening with HPV DNA testing for oncogenic genotypes has been already endorsed as the recommended practice by the ASCCP and other scientific bodies worldwide [16]. One important dividend of HPV testing is the ability to use self-collected samples, which have the potential to increase cover- age of cervical cancer screening to remote areas or to women who are not directly reached by primary healthcare in urban areas. In addition to the above advantages that would come from a change in the screening paradigm from cytology to HPV testing it is also plausible to assume that the latter will serve the needs of cervical cancer screening in the post-vaccination era more efficiently than cytology [17].

Besides cytology, novel triage tests of HPV-positive women include slide assessment based on combined detection of the p16INK4a and Ki-67 biomarker protein expression in LBC specimens, HPV genotyping, and the use of markers including DNA methylation and viral load [17].

Irrespective of the screening strategy, organized vaccination and screening programs with good record keeping are necessary to optimize the future control of cervical cancer [12]. The importance of the principle of prevention equity has been stressed several years ago; Massad et al pointed that alterations in screening frequency will be justified only if vaccination uptake is evenly distributed across social and economic strata or if clinicians can reliably identify adequately vaccinated women for less intensive screening. If cultural or other differences result in the preferential adoption of vaccination by low risk women while rural, minority, and poor women at highest risk reject it, attenuating screening after reaching a threshold level of adoption in the population may paradoxically increase cervical cancer rates. Careful monitoring of vaccine acceptance by age cohorts will be important as policy planners continue to consider the implications of HPV vaccination on cervical cancer prevention strategies [3].

Concerns that vaccinated cohorts will default cervical screening have not been yet corroborated. HPV-vaccination has been so far associated with equal or higher attendance to cervical screening in Sweden in a cohort of opportunistically vaccinated young women [18]. Other authors illustrated that women of an underserved US population who received HPV vaccination closer to their 21 anniversary, and not early in their 14's, were more likely to participate in cervical cancer screening. These authors illustrated that women vaccinated with three doses and screened at or after 21 years had the highest screening rate of $84 \%$ predicting a six-fold increase in screening participation over no vaccine received [19].

Projecting for the following years in cervical cancer prevention, besides established stakeholders, one could foresee an all too important role for new partners; namely screenologists, vaccinologists and definitely policy makers. Screening efforts will be likely focused on high-risk groups such as the immunocompromised, while a role in extended screening intervals for specific age-groups is very likely. Finally, screening in vaccinated girls will most probably be biomarkerbased, with validated HPV DNA assays being the obvious candidates.

\section{Competing Interests}

The authors declare that they have no competing interests.

\section{References}

1. Denny L (2012) Cervical cancer prevention: new opportunities for primary and secondary prevention in the 21st century. Int J Gynaecol Obstet 119 Suppl 1: S80-84.

2. Garnett GP, Kim JJ, French K, Goldie SJ (2006) Chapter 21: Modelling the impact of HPV vaccines on cervical cancer and screening programmes. Vaccine 24 Suppl 3: S3/178-186.

3. Massad LS, Einstein M, Myers E, Wheeler CM, Wentzensen N, et al. (2009) The impact of human papillomavirus vaccination on cervical cancer prevention efforts. Gynecol Oncol 114: 360-364.

4. Lees BF, Erickson BK, Huh WK (2016) Cervical cancer screening: evidence behind the guidelines. Am J Obstet Gynecol 214: 438-443.

5. Katki H, Schiffman M, Castle P, Fetterman B, Poitras N, et al. (2013) Fiveyear risk of $\mathrm{CIN} \mathrm{3+}$ to guide the management of women aged 21 to 24 years. J Low Genit Tract Dis 17: S64-S68.

6. Sigurdsson K (2010) Cervical cancer: cytological cervical screening in Iceland and implications of HPV vaccines. Cytopathology 21: 213-222.

7. Sasieni P, Adams J, Cuzick J (2003) Benefit of cervical screening at different ages: evidence from the UK audit of screening histories. $\mathrm{Br} J$ Cancer 89: 88-93.

8. Stanley M (2008) Human papillomavirus vaccines versus cervical cancer screening. Clin Oncol (R Coll Radiol) 20: 388-394.

9. Herbert A, Holdsworth G, Kubba AA (2008) Cervical screening: why young women should be encouraged to be screened. J Fam Plann Reprod Health Care 34: 21-25.

10. Sopina E, Ashton T (2011) Cost-effectiveness of a cervical screening program with human papillomavirus vaccine. Int J Technol Assess Health Care 27: 290-297.

11. Bosch X, Harper D (2006) Prevention strategies of cervical cancer in the HPV vaccine era. Gynecol Oncol 103: 21-24.

12. Lynge E, Antilla A, Arbyn M, Segnan N, Ronco G (2009) What's next? Perspectives and future needs of cervical screening in Europe in the era of molecular testing and vaccination. Eur J Cancer 45: 2714-2721.

13. Almonte M, Sasieni P, Cuzick J (2011) Incorporating human papillomavirus testing into cytological screening in the era of prophylactic vaccines. Best Pract Res Clin Obstet Gynaecol 25: 617-29.

14. Bosch FX, Robles C, Díaz M, Arbyn M, Baussano I, et al. (2016) HPVFASTER: broadening the scope for prevention of HPV-related cancer. Nat Rev Clin Oncol 13: 119-132. 
Citation: Michail G, Androutsopoulos G, Decavalas G (2016) Cervical Cancer Surveillance - Considerations in the Vaccination Era. Int J Community Fam Med 1: 117. doi: https://doi.org/10.15344/2456-3498/2016/117

Page 3 of 3

15. Jeronimo J, Bansil P, Valdez M, Kang L, Zhao F, et al. (2015) The Influence of Human Papillomavirus Genotypes on Visual Screening and Diagnosis of Cervical Precancer and Cancer. J Low Genit Tract Dis 19: 220-223.

16. Huh W, Ault K, Chelmow D, Davey D, Goulart R, et al. (2015) Use of primary high-risk human papillomavirus testing for cervical cancer screening: interim clinical guidance. Obstet Gynecol 125: 330-337.

17. El-Zein M, Richardson L, Franco EL (2016) Cervical cancer screening of HPV vaccinated populations: Cytology, molecular testing, both or none. J Clin Virol 76 Suppl 1: S62-68

18. Herweijer E, Feldman A, Ploner A, Arnheim-Dahlstrom L, Uhnoo I, et al (2015) The Participation of HPV-Vaccinated Women in a National Cervica Screening Program: Population-Based Cohort Study. PLoS One 10: e0134185

19. Paynter C, Van Treeck B, Verdenius I, Lau A, Dhawan T, et al. (2015) Adherence to cervical cancer screening varies by human papillomavirus vaccination status in a high-risk population. Prev Med Rep 2: 711-716. 